\title{
Cytoplasmic Keap1 Expression Is Associated With Poor Prognosis in Endometrial Cancer
}

\author{
ANNE M. AHTIKOSKI ${ }^{1}$, JUHA KANGAS ${ }^{2}$, RIIKKA SALONEN ${ }^{1}$, ULLA PUISTOLA $^{2}$ and PEETER KARIHTALA ${ }^{3}$ \\ ${ }^{1}$ Cancer and Translational Medicine Research Unit, Department of Pathology, \\ Oulu University Hospital, University of Oulu, Oulu, Finland; \\ ${ }^{2}$ Department of Gynecology and Obstetrics, Pedego Research Unit, \\ Oulu University Hospital, University of Oulu, Oulu, Finland; \\ ${ }^{3}$ Department of Oncology and Radiotherapy, Medical Research Center Oulu, \\ Oulu University Hospital, University of Oulu, Oulu, Finland
}

\begin{abstract}
Background/Aim: Oxidative stress is involved in several carcinogenic pathways. Nuclear factor erythroid 2-related factor (Nrf2), Kelch-like ECH-associated protein 1 (Keapl) and Park7 (DJ-1) are the main regulators of antioxidant enzymes eliminating reactive oxidative species (ROS). The roles of these proteins were studied as potential prognostic factors in endometrial cancer. Materials and Methods: Nrf2, Keap1 and DJ-1 expression in endometrial carcinomas was analyzed immunohistochemically. Correlations between staining patterns and clinical prognostic variables were evaluated. Results: Extensive cytoplasmic Keapl staining correlated to several factors associated with poor prognosis of endometrial cancer including advanced stage, poor histological differentiation, lymphovascular invasion, pelvic lymph node metastasis and deep myometrial invasion. In multivariate analysis, cytoplasmic Keapl was a stronger predictor of poor progression-free survival than grade. Nuclear Nrf2 staining was seen in all patients with lymph node metastasis while DJ-1 staining was associated with clinically favourable disease types. Conclusion: Cytoplasmic Keapl expression indicates poor prognosis in endometrial cancer.
\end{abstract}

Endometrial cancer is the most common gynecological cancer in developed countries (1). The majority (90\%) of women diagnosed with endometrial cancer are older than 50 years. Five-year survival (including all stages) is excellent, but in cases of advanced or high-risk-early-stage disease it is less than $17 \%$ (2). Obesity, diabetes mellitus, hypertension,

Correspondence to: Anne Ahtikoski, MD, Ph.D., Department of Pathology, P.O. Box 50, 90029 Oulu University Hospital, Finland. Tel: +358 401347325, Fax: +358 83152177, e-mail: anne.ahtikoski@oulu.fi

Key Words: Nrf2, Keap1, DJ-1, oxidative stress, endometrial cancer. late menopause, hyperestrogenism, nulliparity, infertility and genetic predisposition have all been identified as risk factors of endometrial cancer (3). Endometrial carcinoma is traditionally divided into two types based on histopathology and pathogenesis. Type I includes histological grade I and II endometrioid carcinomas and mucinous carcinomas whereas type II includes clear cell, serous, grade III endometrioid and mixed carcinomas. Over $80 \%$ of endometrial carcinomas are of type I.

Oxidative stress is a dynamic condition where the amount of reactive oxygen species (ROS) and the antioxidant defence mechanisms are imbalanced (4). Oxidative stress has been proven to be involved in carcinogenesis and prognosis in various malignant and premalignant diseases. There are also various defence mechanisms to counteract the unfavourable effects of ROS (5).

Nuclear factor erythroid 2 (NF-E2)-related factor 2 (Nrf2) has become a subject of widespread interest in cancer research. The Nrf2-regulated pathway can be considered as one of the most effective ways of a cell to protect itself against harmful ROS (6). When activated, Nrf2 binds to an antioxidant response element (ARE) together with Maf proteins and induces genes for several enzymes, metalbinding proteins, molecular chaperones and drug transporters which are involved in antioxidant defence mechanisms $(7,8)$. Under physiological conditions almost all Nrf2 is located in the cytoplasm. Kelch-like ECH-associated protein 1 (Keap1) is a protein that binds to Nrf2 and via the ubiquitin proteasome pathway represses it. Under oxidative stress Nrf2Keap1 interaction is disturbed, which leads to up-regulation of Nrf2 and its accumulation in the nucleus (9). This may help cancer cells to survive by protecting them from oxidative damage caused by chemotherapeutic drugs (10).

DJ-1 (PARK7) is a multifunctional proto-oncogenic protein that identifies oxidative damage. It prevents binding of Keap1 to Nrf2 and activates several antioxidant genes 
such as Nrf2-regulated antioxidant enzyme NAD $(\mathrm{P}) \mathrm{H}$ dependent quinone oxidoreductase 1 (NQO1) (11). Elevated DJ-1 levels have been found in various cancers such as leukemias, astrocytomas and renal carcinomas (12-14). Notable expression of Nrf2 and DJ-1 has also been seen in non-small-cell lung cancer, where it seems to be associated with poor prognosis (15).

This study aimed to evaluate the prognostic value of the levels of Nrf2, Keap1 and DJ-1 in endometrial carcinoma.

\section{Materials and Methods}

The study population consisted of 80 patients diagnosed with endometrial carcinoma: 71 endometrioid carcinomas, 7 serous carcinomas and 2 mixed carcinomas. Tissue samples had been collected in 2003-2011 and were stored at the Department of Pathology, Oulu University Hospital. FIGO classification 2009 was used to determine the stage of the disease. Clinical data were gathered from the patient records of Oulu University Hospital (Table I).

Tissue sections $(3.5 \mu \mathrm{m})$ in paraffin wax were used for immunohistochemical staining. They were deparaffinized in xylene for $3 \times 3 \mathrm{~min}$, rehydrated in a descending series of alcohol solutions and then rinsed. Antigen retrieval was completed in citrate buffer solution ( $\mathrm{pH} 6$ ) in a microwave oven ( $800 \mathrm{~W} 2 \mathrm{~min}, 150 \mathrm{~W} 2 \mathrm{~min})$ followed by cooling at room temperature for $20 \mathrm{~min}$. Neutralization of endogenous peroxidase activity was carried out in peroxidase arrest solution (Dako, Glostrup, DK).

The antibodies used were rabbit monoclonal anti-Nrf2 (EP1808Y, Abcam, Cambridge, UK) at 1:300 dilution, goat polyclonal antiKeap1 (Santa Cruz Biotechnology, Santa Cruz, USA) at 1:100 dilution and rabbit polyclonal anti-PARK7/DJ-1 (Abcam, Cambridge, UK) at 1:10,000 dilution. Dako Envision Kits (Dako, Glostrup, DK) were used for the detection of Nrf2 and DJ-1 antibodies, whereas goat-on-rodent HRP-polymer kit (Biocare GHP516L, Biocare Medical, Concord, CA, USA) was used for Keap1.

Quantitation of staining was performed in both cytoplasm and nuclei of the neoplastic tissues. The extent of moderate or strong staining was defined as: $0=0-5 \% ; 1=6-100 \%$.

Statistical analysis. Statistical analyses were carried out with SPSS 21.0 for Windows software. Fisher's two-sided exact test was used to compare the clinical data and immunohistochemical expression. Kaplan- Meier curves with the log-rank test were applied in the progression-free survival analysis. Overall survival analysis was not produced due to the insufficient number of events during follow up. $p$-Values were considered statistically significant when $p<0.05$. Cox regression analysis was applied in multivariate analysis, where the most important traditional prognostic factors, i.e. stage (stage I - II or stage III) and grade (low-grade or high-grade) were included in the model.

\section{Results}

Moderate to strong cytoplasmic Keap1 expression (>5\%) in malignant epithelium was found in 37 samples $(46.3 \%)$ (Table II, Figure 1). Cytoplasmic Keap1 was associated with more advanced disease (stage II-III), high grade tumors, non-
Table I. Clinicopathological data of 80 subjects.

\begin{tabular}{lcc}
\hline & \multicolumn{2}{c}{ Mean (range) } \\
\hline Age at diagnosis & \multicolumn{2}{c}{$65(48-86)$} \\
BMI $\left(\mathrm{kg} / \mathrm{m}^{2}\right)(\mathrm{n}=75)$ & \multicolumn{2}{c}{$28.8(18.4-42.6)$} \\
\hline Carcinoma type & $\mathrm{n}$ & $\%$ \\
\hline Endometrioid & 71 & $88.8 \%$ \\
$\quad$ Serous & 7 & $8.8 \%$ \\
Mixed & 2 & $2.5 \%$ \\
FIGO stage & & \\
Ia & 30 & $37.5 \%$ \\
Ib & 27 & $33.8 \%$ \\
II & 9 & $11.3 \%$ \\
IIIa & 3 & $3.8 \%$ \\
IIIc & 11 & $13.8 \%$ \\
Histological grade & & \\
Low-grade & 64 & $80 \%$ \\
High-grade & 16 & $20 \%$ \\
& & \\
Myometrial invasion $>50 \%$ & 44 & $55.0 \%$ \\
Lymphovascular invasion & 41 & $51.3 \%$ \\
Recurrence & 16 & $20.0 \%$ \\
Deaths & 6 & $7.5 \%$ \\
\end{tabular}

endometrioid histology, lymphovascular invasion, pelvic lymph-node metastasis and deep myometrial invasion. The presence of cytoplasmic Keap1 expression was a predictor of poor progression-free survival (PFS) $(p=0.011)$ (Figure $2)$. When stage, grade and cytoplasmic Keap1 expression were all included in Cox regression analysis, none of these factors remained significant, probably as a result of the limited number of endpoints. However, when only grade and Keap1 expression were included in the model, cytoplasmic Keap1 was a more significant predictor of poor PFS (relative risk $(\mathrm{RR})=3.423 ; 95 \% \mathrm{CI}=1.030-11.372 ; p=0.045)$ than grade $(\mathrm{RR}=1.610 ; 95 \% \mathrm{CI}=0.522-4.969 ; p=0.408)$. Nuclear Keap1 expression was mainly seen in neoplastic epithelium and only a few samples had positive staining in areas with epithelial hyperplasia. Nuclear Keap1 expression was seen only in 4 samples $(4.0 \%)$, while 76 samples $(95.0 \%)$ were negative (Figure 1). In contrast to cytoplasmic expression, nuclear Keap1 was not associated with any of the other clinicopathological parameters.

Cytoplasmic Nrf2 expression in malignant epithelium was seen in 63 samples $(78.8 \%$ ) (Table II). Moderate or strong nuclear Nrf2 expression was seen in $54(67.5 \%)$ of the samples and, in particular this staining pattern was recorded in all patients with pelvic lymph-node metastasis (9/9) (Figure 1). Expression of Nrf2 was not associated with any other clinical parameter. Nuclear DJ-1 expression (Figure 1) was observed in 39 samples (48.0\%) (Table II). It was associated with low- 
Table II. Number of cases showing moderate to strong (>5\%) cytoplasmic or nuclear immunostaining in neoplastic epithelium. Significant (and nearly significant) $p$-values are presented.

\begin{tabular}{|c|c|c|c|c|c|c|c|}
\hline & \multicolumn{3}{|c|}{ Keap1 } & \multicolumn{2}{|l|}{ Nrf2 } & DJ-1 & \multirow{2}{*}{$\frac{\text { Total }}{\mathrm{n}(\%)}$} \\
\hline & $\begin{array}{c}\text { Cytoplasmic } \\
\text { n (\%) }\end{array}$ & $\begin{array}{l}\text { Nuclear } \\
\text { n (\%) }\end{array}$ & $\begin{array}{c}\text { Cytoplasmic } \\
\text { n }(\%)\end{array}$ & $\begin{array}{l}\text { Nuclear } \\
\text { n (\%) }\end{array}$ & $\begin{array}{c}\text { Cytoplasmic } \\
\text { n }(\%)\end{array}$ & $\begin{array}{c}\text { Nuclear } \\
\text { n }(\%)\end{array}$ & \\
\hline \multicolumn{8}{|l|}{ Histological type } \\
\hline Endometrioid & $28(39.4)$ & $2(2.8)$ & $54(77.1)$ & $46(65.7)$ & $56(83.6)$ & $36(53.7)$ & $71(88.8)$ \\
\hline Non-endometrioid & $9(100) p=0.0005$ & $2(22.2)$ & $9(100)$ & $8(88.9)$ & $7(87.5)$ & $3(37.5)$ & $11(11.3)$ \\
\hline \multicolumn{8}{|l|}{ Grade } \\
\hline Low-grade & $23(35.9)$ & $2(3.1)$ & $49(77.8)$ & $42(66.7)$ & $50(83.3)$ & $33(55.0)$ & $64(80.0)$ \\
\hline High-grade & $14(87.5) p=0.00023$ & $2(12.5)$ & $14(87.5)$ & $12(75.0)$ & $13(86.7)$ & $6(40.0)$ & $16(20.0)$ \\
\hline \multicolumn{8}{|l|}{ Stage } \\
\hline Stage I & $17(29.8)$ & $2(3.5)$ & $45(80.4)$ & $37(66.1)$ & $43(81.1)$ & $30(56.6)$ & $57(71.3)$ \\
\hline Stage II-III & $20(87.0) p=0.000036$ & $2(8.7)$ & $18(78.3)$ & $17(73.9)$ & $20(90.9)$ & $9(40.9)$ & $23(28.7)$ \\
\hline \multicolumn{8}{|c|}{ Myometrial invasion $>50 \%$} \\
\hline Yes & $25(56.8) p=0.044$ & $1(2.3)$ & $34(77.3)$ & $28(63.6)$ & $35(83.3)$ & $15(35.7)$ & $44(55.0)$ \\
\hline No & $12(33.3)$ & $3(8.3)$ & $29(82.9)$ & $26(74.3)$ & $28(84.8)$ & $24(72.7) p=0.0022$ & $36(45.0)$ \\
\hline \multicolumn{8}{|c|}{ Lymphovascular invasion } \\
\hline Yes & $25(61.0) p=0.0080$ & $2(4.9)$ & $34(85.0)$ & $24(61.5)$ & $33(82.5)$ & $16(40.0)$ & $41(51.2)$ \\
\hline No & $12(30.8)$ & $2(5.1)$ & $29(74.4)$ & $30(75.0)$ & $30(85.7)$ & $23(65.7) p=0.026$ & $39(48.8)$ \\
\hline \multicolumn{8}{|l|}{$\begin{array}{l}\text { Pelvic lymph } \\
\text { node metastasis }\end{array}$} \\
\hline Yes & $8(88.9) p=0.0093$ & $3(5.7)$ & $9(100)$ & $9(100)(p=0.051)$ & $6(75.0)$ & $1(12.5)$ & $9(14.5)$ \\
\hline No & $21(39.6)$ & $1(11.1)$ & $39(75.0)$ & $35(67.3)$ & $43(86.0)$ & $25(50.0)$ & $53(85.5)$ \\
\hline
\end{tabular}

depth myometrial invasion and absence of lymphovascular invasion. Cytoplasmic DJ-1 positivity was seen in 63 samples (84\%) (Figure 1). No statistically significant correlations were found when the expression patterns of Keap1, Nrf 2 and DJ-1 were compared with each other.

\section{Discussion}

Patients with early-stage endometrioid-type endometrial carcinomas have a relatively good prognosis, with 10 -year overall survival being $80 \%$ on average (16). However, there is still a certain subgroup of patients whose disease relapses. This has raised an urgent need to find some additional prognostic factors for clinical decision-making and for treatment tools. One very promising prognostic marker protein for endometrial cancer is the L1-cell adhesion molecule (L1CAM; CD171), the expression of which in early-stage endometrial carcinoma patients has indicated a more aggressive behaviour of the disease (17). Recently, genomic classification of endometrial cancer has also revealed new prognostic factors in endometrial cancer. This study aimed to find additional prognostic markers to further evaluate factors connected to the behavior of endometrial cancer. ARE -regulation was evaluated by assessing the expression patterns of Nrf2, Keap1 and DJ-1. The staining patterns were compared in relation to clinicopathological features such as tumor size, histological grade, pelvic lymph- node metastasis, myometrial invasion, lymphovascular invasion and relapse-free survival.

Although Nrf2 has been proven to have protective effects against ROS under physiological conditions, it can also provide proliferation advantage to malignant cells during carcinogenesis. The Nrf2-induced ARE-dependent defence mechanism protects cells under oxidative stress. Nrf2 also provides a growth advantage to malignant cells by inducing the expression of genes that are involved in $\mathrm{NAD}(\mathrm{P}) \mathrm{H}$ regeneration and also the genes that regulate cellular glucose influx and purine generation (18). High levels of Nrf2 expression have been found in type II endometrial carcinomas, which are known to develop chemoresistance (10). In addition, nuclear expression of Nrf2 is associated with poor survival in pancreatic adenocarcinoma, non-smallcell lung cancer, melanoma and gliomas (19-22). Activation of the Nrf2-Keap1-ARE pathway is also related to radiation resistance in lung-cancer patients (23). According to earlier studies, Nrf2 is expressed in most tumors in our study. Interestingly all endometrial carcinomas with lymph node metastasis were positive for Nrf2. Even though sensitivity of $\mathrm{Nrf} 2$ as a marker for lymph node metastasis is not high, this finding justifies further studies on the role of $\mathrm{Nrf} 2$ as a possible specific marker for advanced tumor stage.

Keap1 was associated with all traditional prognostic factors of endometrial cancer and also an unfavorable PFS. According to our results, elevated expression of cytoplasmic 

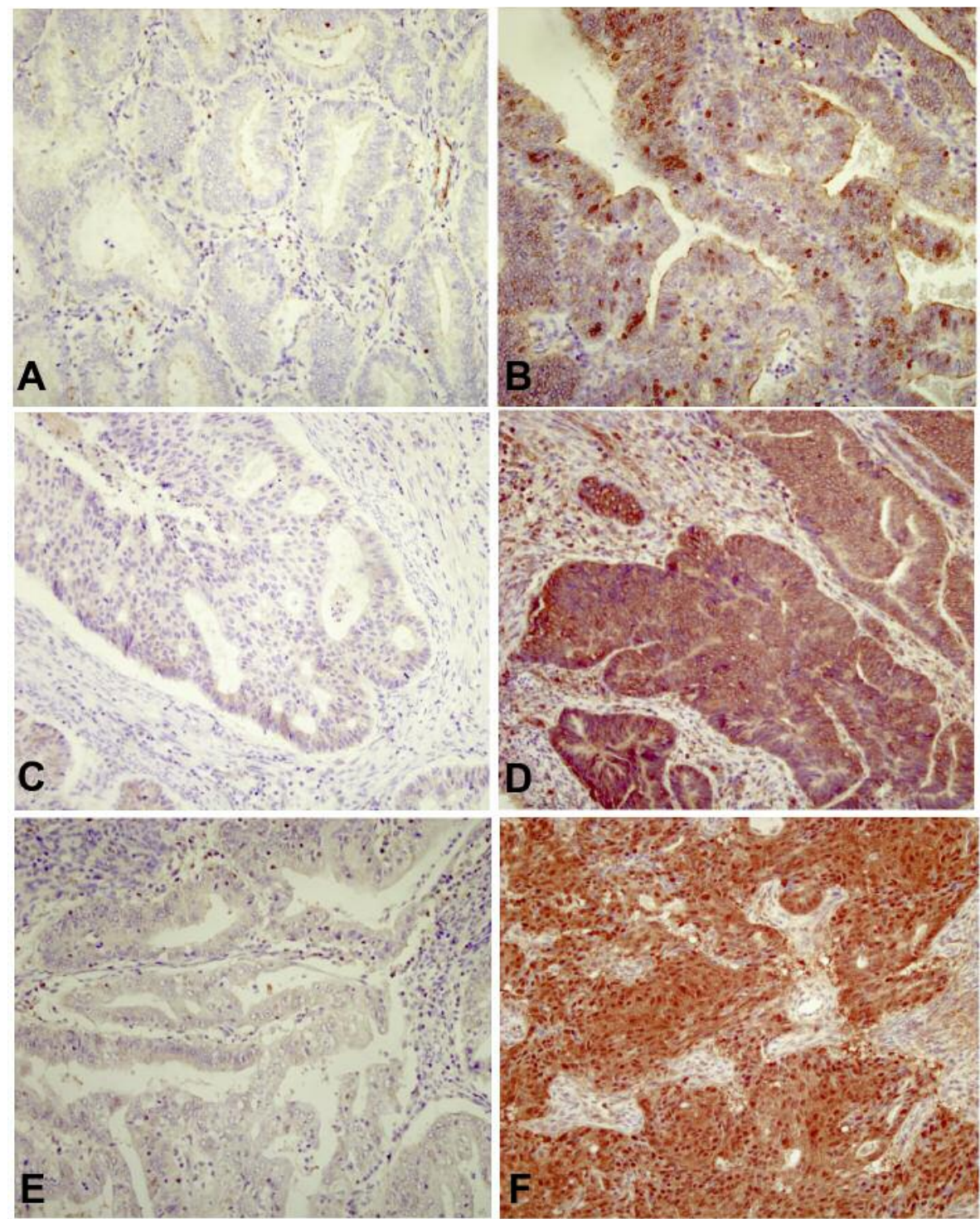

Figure 1. Expression of Keapl (A-B), Nrf2 (C-D) and DJ-1 (E-F) in endometrial carcinoma. Cytoplasmic Keapl staining was stronger in highgrade $(B)$ versus low-grade carcinoma (A). Stronger Nrf2 staining was observed in carcinomas with nodal metastasis $(D)$ compared to carcinomas without metastasis $(C)$. Cytoplasmic DJ-1 expression was observed in most carcinomas yet nuclear staining $(F)$ was associated to less advanced tumor stage. Some carcinomas showed no DJ-1 staining (E).

Keap1 is correlated to more advanced disease and invasiveness of the tumor. Correspondingly, elevated Keap1 expression has been associated with a triple-negative phenotype in breast cancer as well as worse survival when patients with estrogen receptor-positive tumours were also taken into account (24). Although Keap1 is considered to be the main regulator of $\mathrm{Nrf2}$, there are also other factors involved in regulating Nrf2 such as, protein kinase C, Jun Nterminal kinase and phosphatidylinositol kinase (25). It is probable that Keap1 overexpression reflects Keap1 induction, particularly in the most oxidatively stressed tumors. However, our results suggest that Keap1 could be a beneficial prognostic factor in endometrial cancer and in designing adjuvant treatment.

DJ-1 is considered to be a proto-oncogene due to its ability to protect cells against oxidative stress-derived damage by preventing apoptosis (26). Tumor invasiveness and metastatic potential are thought to be associated with epithelial-mesenchymal transition (EMT) generated by suppression of PTEN by DJ-1 (27). Overexpression of DJ-1 has been found in various malignant diseases. It has been linked to invasion and tumor metastasis in nasopharyngeal 


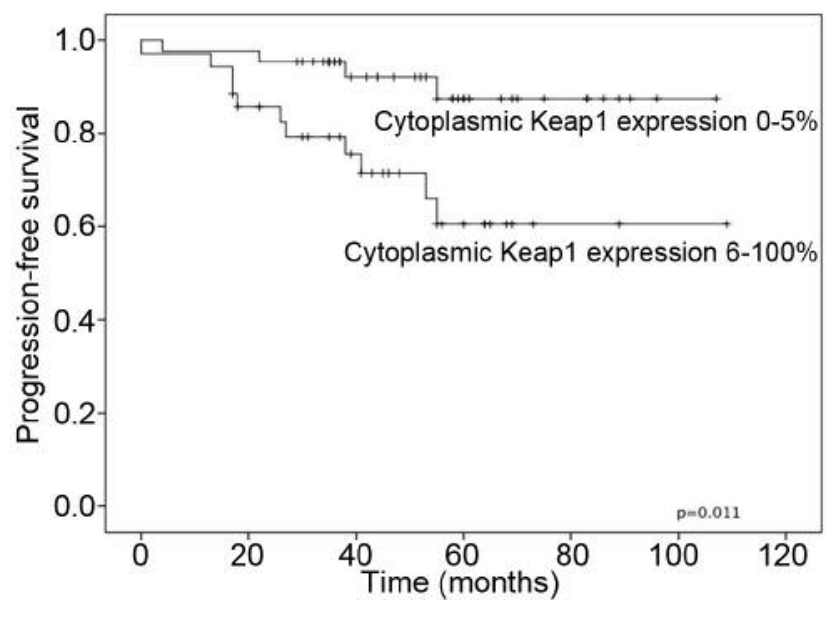

Figure 2. Kaplan-Meier curves comparing low and high cytoplasmic Keap1 expression.

carcinoma and non-small-cell lung-cancer $(28,29)$. Nuclear expression of DJ-1 has also been found in lung-cancer patients with distant metastases (15). Some recent studies have reported a higher immunoscore in endometrial serous carcinoma versus endometrioid carcinoma (30) and increased DJ-1 serum level especially in serous carcinoma $(30,31)$. In our study, elevated nuclear DJ-1 was associated with the absence of lymphovascular invasion and with lower myometrial invasion. Therefore, it seems that the observed association of DJ-1 with lower stage endometrial cancer gives new perspective to the research field and suggests that the role of DJ-1 as a predictor of unfavourable prognosis is not as clear.

In conclusion, the ARE signalling route in oxidative stress seems to be activated and to be involved in the pathogenesis of endometrial cancer. Clinically the most relevant marker of unfavourable prognosis seems to be cytoplasmic Keap1 expression. Yet, further studies are needed to establish the possible role of Keap1 in clinical decision making. Interestingly, in contradiction to earlier studies with endometrial and other malignancies, DJ-1 was not associated with an aggressive tumor behaviour. Hence, the role of DJ-1 in carcinogenesis is not yet revealed, but needs further study.

\section{Conflicts of Interest}

The Authors have stated no conflicts of interest in connection to this article.

\section{Authors' Contributions}

Ulla Puistola and Peeter Karihtala contributed to experimental design and conception as well as revision of the manuscript; Juha Kangas and Riikka Salonen contributed to clinical data collection;
Anne Ahtikoski and Juha Kangas contributed to histopathological evaluation and data collection; Peeter Karihtala and Juha Kangas contributed to data analysis; Anne Ahtikoski and Juha Kangas contributed to writing of the manuscript.

\section{Acknowledgements}

This study was supported financially by a state subsidy granted to the University Hospital of Oulu. Riitta Vuento and Manu Tuovinen are acknowledged for their contribution to the laboratory work.

\section{References}

1 Colombo N, Creutzberg C, Amant F, Bosse T, González-Martín A, Ledermann J, Marth C, Nout R, Querleu D, Mirza MR and Sessa C; ESMO-ESGO-ESTRO Endometrial Consensus Conference Working Group: ESMO-ESGO-ESTRO Consensus Conference on Endometrial Cancer: Diagnosis, Treatment and Follow-up. Int J Gynecol Cancer 26: 2-30, 2015. PMID: 26645990, DOI: 10.1097/IGC.0000000000000609

2 Jemal A, Siegel R, Xu J and Ward E: Cancer statistics, 2010. CA Cancer J Clin 60: 277-300, 2010. PMID: 20610543, DOI: $10.3322 /$ caac. 20073

3 Colombo N, Preti E, Landoni F, Carinelli S, Colombo A, Marini $\mathrm{C}$ and Sessa C; ESMO Guidelines Working Group: Endometrial cancer: ESMO Clinical Practice Guidelines for diagnosis, treatment and follow-up. Ann Oncol 24: vi33-8, 2013. PMID: 24078661, DOI: 10.1093/annonc/mdt353

4 Karihtala $\mathrm{P}$ and Soini Y: Reactive oxygen species and antioxidant mechanisms in human tissues and their relation to malignancies. APMIS 115: 81-103, 2007. PMID: 17295675, DOI: $10.1111 / \mathrm{j} .1600-0463.2007 . a p m \_514 . x$

5 Karihtala P and Puistola U: Hypoxia and oxidative stress in the pathogenesis of gynecological cancers and in therapeutical options. Curr Cancer Ther Rev 7: 37-55, 2011. PMID: 21693047, DOI: 10.1186/1471-2407-11-262

6 Thimmulappa RK, Mai KH, Srisuma S Kensler TW, Yamamoto $\mathrm{M}$ and Biswal S: Identification of Nrf2-regulated genes induced by the chemopreventive agent sulforaphane by oligonucleotide microarray. Cancer Res 62(18): 5196-5203, 2002. PMID: 12234984

7 Itoh K, Mimura $\mathrm{J}$ and Yamamoto M: Discovery of the negative regulator of Nrf2, Keap1: a historical overview. Antioxid Redox Signal 13(11): 1665-1678, 2010. PMID: 20446768, DOI: 10.1089/ars.2010.3222

$8 \mathrm{Hu}$ R, Saw CL, Yu R and Kong AN: Regulation of NF-E2related factor 2 signaling for cancer chemoprevention: antioxidant coupled with anti-inflammatory. Antioxid Redox Signal 13(11): 1679-1698, 2010. PMID: 20486765, DOI: 10.1089/ars.2010.3276

9 Taguchi K, Motohashi $\mathrm{H}$ and Yamamoto $\mathrm{M}$ : Molecular mechanisms of the Keap1-Nrf2 pathway in stress response and cancer evolution. Genes Cells 16(2): 123-140, 2011. PMID: 21251164, DOI: 10.1111/j.1365-2443.2010.01473.x

10 Jiang T, Chen N, Zhao F, Wang XJ, Kong B, Zheng W and Zhang DD: High levels of Nrf2 determine chemoresistance in type II endometrial cancer. Cancer Res 70(13): 5486-5496, 2010. PMID: 20530669, DOI: 10.1158/0008-5472.CAN-100713 
11 Clements CM, McNally RS, Conti BJ, Mak TW and Ting JP: DJ-1, a cancer- and Parkinson's disease-associated protein, stabilizes the antioxidant transcriptional master regulator Nrf2. Proc Natl Acad Sci USA 103(41): 15091-15096, 2006. PMID: 17015834, DOI: 10.1073/pnas.0607260103

12 Liu H, Wang M, Li M, Wang D, Rao Q, Wang Y, Xu Z and Wang J: Expression and role of DJ-1 in leukemia. Biochem Biophys Res Commun 375(3): 477-483, 2008. PMID: 18722352, DOI: 10.1016/j.bbrc.2008.08.046

13 Miyajima Y, Sato Y, Oka H, Utsuki S, Kondo K, Tanizaki Y, Nagashio R, Tsuchiya B, Okayasu I and Fuji K: Prognostic significance of nuclear DJ-1 expression in astrocytoma. Anticancer Res 30(1): 265-269, 2010. PMID: 20150646

14 Sitaram RT, Cairney CJ, Grabowski P, Keith WN, Hallberg B, Ljungberg B and Roos G: The PTEN regulator DJ-1 is associated with hTERT expression in clear cell renal cell carcinoma. Int J Cancer 125(4): 783-790, 2009. PMID: 19384955, DOI: $10.1002 / \mathrm{ijc} .24335$

15 Merikallio H, Paakko P, Kinnula VL, Harju T and Soini Y: Nuclear factor erythroid-derived 2-like $2(\mathrm{Nrf} 2)$ and DJ1 are prognostic factors in lung cancer. Hum Pathol 43(4): 577-584, 2012. PMID: 21943684, DOI: 10.1016/j.humpath.2011.05.024

16 Kitchener HC and Trimble EL: Endometrial Cancer Working Group of the Gynecologic Cancer Intergroup: Endometrial cancer state of the science meeting. Int J Gynecol Cancer 19(1): 134-140, 2009. PMID: 19258955, DOI: 10.1111/IGC.0b013e $3181995 f 90$

17 Zeimet AG, Reimer D, Huszar M, Winterhoff B, Puistola U, Azim SA, Müller-Holzner E, Ben-Arie A, van Kempen LC, Petru E, Jahn S, Geels YP, Massuger LF, Amant F, Polterauer S, Lappi-Blanco E, Bulten J, Meuter A, Tanouye S, Oppelt P, Stroh-Weigert M, Reinthaller A, Mariani A, Hackl W, Netzer M, Schirmer U, Vergote I, Altevogt P, Marth C and Fogel M: L1CAM in early-stage type I endometrial cancer: results of a large multicenter evaluation. J Natl Cancer Inst 105(15): 11421150, 2013. PMID: 23781004, DOI: 10.1093/jnci/djt144

18 Jaramillo MC and Zhang DD: The emerging role of the Nrf2Keap1 signaling pathway in cancer. Genes Dev 27(20): 21792191, 2013. PMID: 24142871, DOI: 10.1101/gad.225680.113

19 Soini Y, Eskelinen M, Juvonen P, Kärjä V, Haapasaari KM, Saarela A and Karihtala P: Nuclear Nrf2 expression is related to a poor survival in pancreatic adenocarcinoma. Pathol Res Pract 210(1): 35-39, 2014. PMID: 24189098, DOI: 10.1016/j.prp. 2013.10.001

20 Solis LM, Behrens C, Dong W, Suraokar M, Ozburn NC, Moran CA, Corvalan AH, Biswal S, Swisher SG, Bekele BN, Minna JD, Stewart DJ and Wistuba II: Nrf2 and Keap1 abnormalities in non-small cell lung carcinoma and association with clinicopathologic features. Clin Cancer Res 16(14): 3743-3753, 2010. PMID: 20534738, DOI: 10.1158/1078-0432.CCR-09-3352

21 Kanamori M, Higa T, Sonoda Y, Murakami S, Dodo M, Kitamura H, Taquchi K, Shibata T, Watanabe M, Suzuki H, Shibahara I, Saito R, Yamashita Y, Kumabe T, Yamamoto M, Motohashi $\mathrm{H}$ and Tominaga T: Activation of the NRF2 pathway and its impact on the prognosis of anaplastic glioma patients. Neuro Oncol 17(4): 555-565, 2015. PMID: 25304134, DOI: $10.1093 /$ neuonc/nou 282
22. Hintsala HR, Jokinen E, Haapasaari KM, Moza M, Ristimäki A, Soini Y, Koivunen J and Karihtala P: Nrf2/Keap1 pathway and expression of oxidative stress lesions 8-hydroxy-2'deoxyguanosine and nitrotyrosine in melanoma. Anticancer Res 36(4): 1497-506, 2016. PMID: 27069125

23 Singh A, Bodas M, Wakabayashi N, Bunz F and Biswal S: Gain of Nrf2 function in non-small-cell lung cancer cells confers radioresistance. Antioxid Redox Signal 13(11): 1627-1637, 2010. PMID: 20446773, DOI: 10.1089/ars.2010.3219

24 Karihtala P, Kauppila S, Soini Y and Jukkola-Vuorinen A: Oxidative stress and counteracting mechanisms in hormone receptor positive, triple-negative and basal-like breast carcinomas. BMC Cancer 11: 262-2407-11-262, 2011. PMID: 21693047, DOI: 10.1186/1471-2407-11-262

25 Lau A, Villeneuve NF, Sun Z, Wong PK and Zhang DD: Dual roles of Nrf2 in cancer. Pharmacol Res 58: 262-270, 2008. PMID: 18838122, DOI: 10.1016/j.phrs.2008.09.003

26 Taira T, Saito Y, Niki T, Iguchi-Ariga SM, Takahashi K and Ariga H: DJ-1 has a role in antioxidative stress to prevent cell death. EMBO Rep 5(2): 213-218, 2004. PMID: 14749723, DOI: 10.1038/sj.embor.7400074

27 Yao Y, Wei H, Liu L, Liu L, Bai S, Li C, Luo Y, Zeng R, Han $\mathrm{M}, \mathrm{Ge} \mathrm{S}$ and $\mathrm{Xu} \mathrm{G}$ : Upregulated DJ-1 promotes renal tubular EMT by suppressing cytoplasmic PTEN expression and Akt activation. J Huazhong Univ Sci Technolog Med Sci 31(4): 469475, 2011. PMID: 21823007, DOI: 10.1007/s11596-011-0475-3

28. Pei XJ, Wu TT, Li B, Tian XY, Li Z and Yang QX: Increased expression of macrophage migration inhibitory factor and DJ-1 contribute to cell invasion and metastasis of nasopharyngeal carcinoma. Int J Med Sci 11(1): 106-115, 2013. PMID: 24396292, DOI: $10.7150 /$ ijms.7264

29 Bai J, Guo C, Sun W, Li M, Meng X, Yu Y, Jin Y, Tong D, Geng J, Huang Q, Qi J and Fu S: DJ-1 may contribute to metastasis of non-small cell lung cancer. Mol Biol Rep 39(3): 2697-2703, 2012. PMID: 21670963, DOI: 10.1007/s11033-011-1024-7

30 Morelli M, Scumaci D, Di Cello A, Venturella R, Donato G, Faniello M, Quaresima B, Cuda G, Zullo F and Costanzo F: DJ1 in endometrial cancer: a possible biomarker to improve differential diagnosis between subtypes. Int J Gynecol Cancer 24: 649-658, 2014. PMID: 24614826, DOI: 10.1097/IGC.0000 000000000102

31 Di Cello A, Di Sanzo M, Perrone FM, Santamaria G, Rania E, Angotti E, Venturella R, Mancuso S, Zullo F, Cuda G and Costanzo F: DJ-1 is a reliable serum biomarker for discriminating high-risk endometrial cancer. Tumor Biol 6: 1-9, 2017. PMID: 28618925, DOI: 10.1177/1010428317705746
Received January 8, 2019

Revised January 16, 2019

Accepted January 18, 2019 